\title{
Transmission Resonance in an Infinite Strip of Phason-Defects of a Penrose Approximant Network
}

\author{
K. Moulopoulos And S. Roche \\ Laboratoire d' Etudes des Propriétés Electronique \\ des Solides, CNRS, 38042 Grenoble
}

\begin{abstract}
An exact method that analytically provides transfer matrices in finite networks of quasicrystalline approximants of any dimensionality is discussed. We use these matrices in two ways: a) to exactly determine the band structure of an infinite approximant network in analytical form; b) to determine, also analytically, the quantum resistance of a finite strip of a network under appropriate boundary conditions. As a result of a subtle interplay between topology and phase interferences, we find that a strip of phason-defects along a special symmetry direction of a low 2-d Penrose approximant, leads to the rigorous vanishing of the reflection coefficient for certain energies. A similar behavior appears in a low 3-d approximant. This type of "resonance" is discussed in connection with the gap structure of the corresponding ordered (undefected) system.
\end{abstract}

PACS numbers: 72.10.-d, 71.10.+x, 61.44.+p 


\section{INTRODUCTION}

Recent experimental work ${ }^{1}$ shows that quasicrystals and quasicrystalline approximants have curious (for metallic materials) transport properties. Anomalously high values of the low-temperature resistivity, and the resistivity decrease with introduction of defects or with increase of temperature, are typical examples. We have recently analyzed ${ }^{2}$ the role of a particular type of phason-defect on the conductance of a Fibonacci chain. From a consideration of the scattering problem of noninteracting electrons through the chain we have demonstrated subtle interference effects between the hyperspace construction and the phase-coherence of the wavefunctions in real space. A full continuous formulation (as opposed to usual discetized approximations of a tight-binding form) was critical ${ }^{2}$ for obtaining these interferences, i.e. the full phase-coherence had to be kept without truncation approximations. Motivated by that work, we here follow a similar continuous scattering formalism, but in model systems of higher dimensionality.

Within a continuous approach, a way to study exactly structural aspects in higher dimensionality, is to treat networks of wires ${ }^{3}$ that form a particular 2-d or 3-d structure. The electrons can propagate only along the wires, but their wavefunctions split at every vertex by respecting continuity and quantum-mechanical current conservation at every vertex. (This splitting is the analog of scattering in the full high-d problem). These are good (exactly soluble) model systems that can show non-trivial interference effects on the

phase of the exact wavefunctions, and that can also demonstrate how these are affected by the particular topology (manifested by the connectivities characterizing the selected structure).

Our goal here is to study such structural effects on phase interferences, but also to see how these are influenced by introduction of defects in the structure, in the form of disruption of long-range order. We find a type of resonance upon introduction of a particular 
kind of defect, which is the result of a subtle interplay between phase interferences and the topology introduced by the defect.

The structures used can be periodic, but with non-trivial unit cells. Periodicity combined with the scattering formalism leads to a natural study of quasicrystalline approximants. We should emphasize that consideration of such a scattering (or equivalently, transmission) problem, gives full information on the states and the spectrum. The band structure of these approximants can be given exactly in closed form (with the coupling of modes in different directions kept in full without approximations). These are therefore good textbook examples of derivations of band structures in high dimensionality, by simply starting from a scattering problem through a single unit cell.

The defects introduced are of a phason-type (but different from the ones studied in ref.2) and in real space they correspond to flips of the internal structure of the unit cell. Our goal is to study the influence of such a flip on the transmission properties. We actually find, as already mentioned, at least one system where the flipping causes a rigorous vanishing of backscattering (reflection coefficient) for some incidence energies. Introduction of an infinite strip of such flippings leads to a "resonance", while introduction of a finite number of them lowers the resistance of an otherwise ordered strip. Although these results are rigorous for a strip of unit cells under some special type of boundary conditions, comparison with the same method but applied to a square lattice gives evidence that this vanishing may be related to creation of some extended states in the infinite lattice upon introduction of flipping. This observation may be seen as a manifestation of anomalous transmission behavior, consistent with numerical works ${ }^{4}$ on 2-d Penrose lattices (in a tight-binding approximation), and also in general agreement with experiments ${ }^{1}$ in real approximants. We also find that the special energies where the resonance occurs lie in the middle of gaps of the infinite (undefected) approximant. We propose this as an evidence that a possible creation of extended states may be due to the creation of levels (upon flipping) in gaps of 
the ordered system.

\section{THE METHOD}

We solve the full continuous and static Schrödinger equation in each branch (wire) of the network. No truncation approximations are made, so that the full phase-coherence of the wavefunctions is maintained. For simplicity, we use a vanishing potential along each wire (free electrons along each branch) and take the length of each branch as common $(l)$ (however we can still form various non-trivial structures by connecting the wires appropriately). For more complicated treatments (i.e. for effects of disorder by introducing random potentials along the wires etc.) the above assumptions can of course be relaxed.

We then match the solutions from all wires that join at a point (vertex): continuity of the wavefunction, and quantum-mechanical current conservation at each vertex are enforced $^{3}$. The method is formally similar to discussions ${ }^{5}$ of the linearized GinzburgLandau equation in superconducting networks.

We give for example the matching equations for the simplest single-vertex arrangements shown in Fig.1:

For case $(a)$ of three wires we have

$$
A_{1} e^{i k l}+B_{1} e^{-i k l}=A_{1}^{\prime}+B_{1}^{\prime}=A_{0}+B_{0}
$$

from the continuity of the wavefunctions, and

$$
A_{1} e^{i k l}-B_{1} e^{-i k l}=A_{1}^{\prime}-B_{1}^{\prime}+A_{0}-B_{0}
$$

as one way to assure quantum-mechanical current conservation. In the above, $A$ and $B$ are the coefficients of the two linearly independent plane waves (with wavevector $k$ ) along each wire. 
A $2 \times 2$ transfer matrix can be defined that describes the scattering of electrons incident at branch 1 and transmitted at branch $1^{\prime}$, while branch 0 is viewed as "transverse" escape of electrons. If for example we look at a situation where electrons are not incident from outside through branch 0 (which is equivalent to setting $B_{0}=0$ ), but they can only escape through this branch (i.e. $A_{0} \neq 0$ ), then elimination of $A_{0}$ from equations (1) and (2) leads to

$$
\left(\begin{array}{l}
A_{1}^{\prime} \\
B_{1}^{\prime}
\end{array}\right)=\frac{1}{2}\left(\begin{array}{cc}
e^{i k l} & -e^{-i k l} \\
e^{i k l} & 3 e^{-i k l}
\end{array}\right) \cdot\left(\begin{array}{c}
A_{1} \\
B_{1}
\end{array}\right)
$$

which defines the $2 \times 2$ transfer matrix for this simplest possible case (the determinant of this matrix is 1 , as expected, from conservation of probability).

It is important to emphasize that the topology of the selected structure (manifested by the corresponding connectivity of the wires) is crucial for the form of the resulting matrices. To show this, let us also determine the $2 \times 2$ transfer matrix associated with case (b) with two transverse channels of Fig.1. For this case, the matching equations are

$$
A_{1} e^{i k l}+B_{1} e^{-i k l}=A_{1}^{\prime}+B_{1}^{\prime}=A_{0}+B_{0}=A_{0}^{\prime}+B_{0}^{\prime}
$$

from the continuity of the wavefunctions, and

$$
A_{1} e^{i k l}-B_{1} e^{-i k l}=A_{1}^{\prime}-B_{1}^{\prime}+A_{0}-B_{0}+A_{0}^{\prime}-B_{0}^{\prime}
$$

from the current conservation. Once again, if we treat branch 1 as the input branch, $1^{\prime}$ as the output branch, and 0 and $0^{\prime}$ as free transverse escapes (i.e. we impose $B_{0}=B_{0}^{\prime}=0$, i.e. no additional input of electrons from the transverse directions, but only output that is naturally determined from the Schrödinger equation), then elimination of $A_{0}$ and $A_{0}^{\prime}$ from equations (4) and (5) leads to

$$
\left(\begin{array}{l}
A_{1}^{\prime} \\
B_{1}^{\prime}
\end{array}\right)=\left(\begin{array}{cc}
0 & -e^{-i k l} \\
e^{i k l} & 2 e^{-i k l}
\end{array}\right) \cdot\left(\begin{array}{l}
A_{1} \\
B_{1}
\end{array}\right) .
$$

Note that the $2 \times 2$ transfer matrix for this case is different from (3), the sole reason being the fact that there is one additional transverse channel compared to the case $(a)$. (However the determinant of (6) is also 1 , as expected). 
We conclude from these trivial (single-vertex) cases, that the topology (connectivity) is very important for the form of the transfer matrices (and hence of any transmission properties, as, for example, the resistance (see below)). The selected topology actually leads to nontrivial forms for the transfer matrices and the transmission properties, as will be discussed below.

It is important to note that even for a complex problem (of many wires and many vertices forming a particular structure) it is always legitimate to choose one wire as the input channel and any other wire as the output one, with all the rest "outside" wires (i.e. lying in the exterior of the chosen unit) treated as transverse escape-channels, irrespective of their number. By then choosing boundary conditions of some type for the coefficients in these transverse wires, we can always obtain a $2 \times 2$ transfer matrix, as above, but, in general, with elements that can be quite complicated functions of $k l$, the form of which depends on the topology of the structure (of both internal and external connectivities) and describes the complete phase-coherence throughout the selected unit. This will be done in the following sections.

Let us first however use the concept of the transfer matrix, as introduced above, to discuss transmission properties. Usually, for a complex system with many channels, one speaks in terms of the multi-channel Landauer formula ${ }^{6}$ in order to define a measure of the resistance. Here however we find it more convenient to focus on the channels that we choose as input and output branches, and to define an effective resistance, even in the presence of the transverse escapes. By making a small modification ${ }^{7}$ to the standard Landauer argument ${ }^{8}$ we find that this effective resistance is given, for spin- $\frac{1}{2}$ electrons, by

$$
\mathcal{R}=\frac{\pi \hbar}{e^{2}} \frac{(R-T+1)}{2(1-R)}
$$

with $R$ and $T$ the (partial) reflection and transmission coefficients resulting from the $2 \times 2$ transfer matrix (as discussed above) of the problem under consideration. It turns out that 
these coefficients are given by the elements of the transfer matrix $(\hat{T})$ as follows:

$$
R=\frac{\left|T_{21}\right|^{2}}{\left|T_{22}\right|^{2}}
$$

and

$$
T=\frac{1}{\left|T_{22}\right|^{2}}
$$

Note that (7) yields the usual Landauer ratio $R / T$ if there are no transverse escapes or if periodicity is enforced in the transverse channels (or generally whenever $R+T=1$ is valid).

The boundary conditions (for the transverse wires) that we use in what follows are of two types: One might be called "free scattering" and correspond to vanishing transverse incidence (as the ones used in the above trivial single-vertex cases), and the other is periodic boundary conditions in the transverse directions. The latter can lead to band structures, as will be seen below. But a link between the two types is expected, as we now discuss in the case of the simplest 2-d structure: a square network.

\section{EXAMPLE: SQUARE LATTICE}

\section{$\underline{\text { A. Transmission Problem }}$}

The basic vertex problem to be solved is shown in Fig.1-d. Matching the solution in all $N$ vertices results in transfer matrices, with forms depending on the boundary conditions.

1) For "free scattering", namely $C_{0}=D_{N}=0$ (no current input transversely, as discussed in previous Section) we obtain the following $2 N \times 2 N$ transfer matrix (for $N \geq 2$ )

$$
\hat{T}=\frac{1}{2 i \sin k l}\left(\begin{array}{cc}
e^{2 i k l} \hat{1}_{N}+e^{2 i k l} \hat{\Omega} & e^{-2 i k l} \hat{1}_{N}+\hat{\Omega} \\
-\hat{1}_{N}-e^{2 i k l} \hat{\Omega} & \left(1-2 e^{-2 i k l}\right) \hat{1}_{N}-\hat{\Omega}
\end{array}\right)
$$


with $\hat{1}_{N}$ denoting the $N \times N$ identity matrix, and with $\hat{\Omega}$ being an $N \times N$ submatrix of the form

$$
\hat{\Omega}=\left(\begin{array}{ccccccc}
0 & -e^{-i k l} & 0 & 0 & 0 & \ldots & 0 \\
-e^{-i k l} & 1 & -e^{-i k l} & 0 & 0 & \ldots & 0 \\
0 & -e^{-i k l} & 1 & -e^{-i k l} & 0 & \cdots & 0 \\
0 & \cdot & \cdot & \cdot & \cdot & \cdot & 0 \\
0 & \cdot & \cdot & \cdot & -e^{-i k l} & \dot{1} & 0 \\
0 & 0 & 0 & \cdot & 0 & -e^{-i k l} & 0 \\
0 & 0 & 0 & \cdot & 0
\end{array}\right)
$$

Hence the total tranfer matrix of a finite piece of a square network of "width" $N$ and "length" $M$ will be a product of $M$ matrices of the form (10). This matrix summarizes the full information of multiple splittings and it can give all the transmission properties of this network under this type of boundary conditions. (We will see below, however, that a simple choice of width $N=2$ and length $M=2$ (equivalent to a single unit cell) is sufficient to exhibit resonance phenomena related to properties of the infinite square network).

2) For periodic boundary conditions along the transverse direction, namely $C_{N}=$ $C_{0} e^{i N \phi}$ and $D_{N}=D_{0} e^{i N \phi}$ we obtain the corresponding $2 N \times 2 N$ result (again for $N \geq 2)$

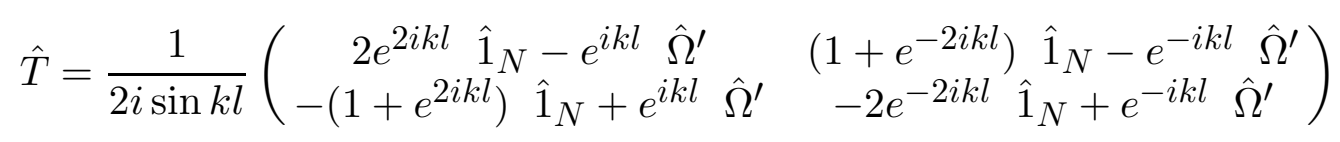

with $\hat{\Omega}^{\prime}$ being again an $N \times N$ submatrix, but now of the form

$$
\hat{\Omega}^{\prime}=\left(\begin{array}{ccccc}
0 & 1 & 0 & \ldots & e^{-i \phi} \\
1 & 0 & 1 & \ldots & 0 \\
0 & 1 & 0 & \ldots & 1 \\
\cdot & . & . & . & . \\
e^{i \phi} & . & 1 & . & . \\
0
\end{array}\right)
$$

and once again the total tranfer matrix of a finite piece of a square network $(N \times M)$ will be a product of $M$ matrices of the form (12).

We make the following important observation for case 1): if we take just a double vertex $(N=2)$, the transfer matrix associated with the unit cell of the square network 
(hence $M=2$ as well) is just the square of matrix (10). This matrix has off-diagonal elements proportional to cot $k l$, which are therefore vanishing for $k l=(2 \rho+1) \frac{\pi}{2}$. These "resonances" that are observed for the single unit cell under "free-scattering" boundary conditions, correspond, as we will show below, to a special property of the bands of the infinite square network and they are related to states that are "maximally extended" (see next Subsection). Hence the finite (even small, with just a single unit cell) transmission problem, for this type of boundary conditions, carries the memory of the most extended states (in the thermodynamic limit) in a form of vanishing of appropriate partial reflection coefficients. This motivates the treatment of a single unit cell of a somewhat more complex network in Section 4.

\section{B. Band Structure}

Let us actually find the exact band structure of an (infinite) square network: This results from relating coefficients periodically in both orthogonal directions. By identifying, for example, in the single-vertex system $(N=M=1)$ (see Fig.1-c) the coefficients in the following way

$$
A_{1}^{\prime}=A_{1} e^{i \phi_{1}}, \quad B_{1}^{\prime}=B_{1} e^{i \phi_{1}}
$$

and

$$
A_{0}^{\prime}=A_{0} e^{i \phi_{2}}, \quad B_{0}^{\prime}=B_{0} e^{i \phi_{2}}
$$

all 8 coefficients appearing in this problem can be eliminated, with a resulting relation between $k l$ and $\phi_{1}$ and $\phi_{2}$. This relation is the exact band structure (since $k$ is related to the energy $E$ by $k^{2}=\frac{2 m E}{\hbar^{2}}$, and the two phases define the crystal momenta $\left(q_{1}\right.$ and $\left.q_{2}\right)$ in the two orthogonal directions through $\phi_{1}=q_{1} l$ and $\phi_{2}=q_{2} l$. The resulting band structure is

$$
\frac{2 m}{\hbar^{2}} E l^{2}=\left(\arccos \left(\frac{1}{2} \cos q_{1} l+\frac{1}{2} \cos q_{2} l\right)\right)^{2}
$$

Plotting (14) for $q_{1} l$ as a function of $E$ (for several fixed values of $q_{2} l$ ), we see gaps 
opening at regions where $q_{1} l$ becomes a complex number. (We also observe a manifestation of the Higgs mechanism (i.e. opening of a gap at $E=0$ ) that also appears and is further discussed in the next Section). It is interesting that, by smoothly changing $q_{2} l$ we see that the band structure is actually moving. This is an additional feature (of coupling between the two modes) in comparison to standard tight-binding band structures. Note, however, that during these movement, the special points $k l=(2 \rho+1) \frac{\pi}{2}$ always lie in bands (and they are the only points that have this property) (see Fig.2). This shows that these points label what could be called "maximally extended" states. Recall that these are the points that give "resonances" (vanishings of the partial reflection coefficients) in the "free-scattering" transfer matrices of a single unit cell. This motivates our later proposition that a resonance that we will find in a non-trivial network, under introduction of a defect, may also be related to possible creation of extended states. (Spectral properties of a more general rectangular lattice has been recently discussed ${ }^{9}$, with emphasis on incommensurability issues with respect to the ratio of the two lattice constants).

\section{A LOW 2-D PENROSE APPROXIMANT}

\section{$\underline{\text { A. Transmission Problem }}$}

The simplest way of filling the plane periodically with the standard two Penrose rhombic tiles is shown in Fig.3-a (where also the two linearly independent directions are shown, labelled by the two phases $\phi_{1}$ and $\phi_{2}$ that will later enter in the band structure). This structure can be viewed as a low 2-d Penrose approximant.

Let us determine the transfer matrix for the scattering of electrons through a single unit cell of such an ordered system, by treating branch 1 as the input branch, and $1^{\prime}$ as the output one (see Fig.3-a)). All other "outside" branches shown in Fig.3-a are treated as 
transverse escapes. Let us therefore apply "free-scattering" boundary conditions for these escape-wires (that are 10 in number). It turns out that we now have overall 30 matching equations (describing the complete physics at the 7 vertices). The total number of branches is 21 (which means 42 coefficients overall), and since from the boundary conditions 10 of them vanish, we can solve this linear system of equations for $(42-10)-30=2$ quantities (i.e. $A_{1}^{\prime}$ and $B_{1}^{\prime}$ ), a procedure which indeed yields a $2 \times 2$ transfer matrix (linearly relating $A_{1}^{\prime}, B_{1}^{\prime}$ with $\left.A_{1}, B_{1}\right)$ as claimed earlier. The above procedure is actually equivalent to inverting a $30 \times 30$ matrix, in order to obtain the final $2 \times 2$ transfer matrix for this problem. This $30 \times 30$ matrix carries the full memory of the structure of the unit cell (through the particular connectivities), both the internal structure, but also the external connectivities as well (recall from earlier discussion in Section 2 that this was crucial for the form of the resulting matrix).

The transfer matrix that results from this procedure gives, through equations (8) and (9), the following exact results for the reflection and transmission coefficients

$$
R=\frac{1}{9} \frac{31913+39744 \cos 2 k l+8343 \cos 4 k l}{5897+6336 \cos 2 k l+567 \cos 4 k l}
$$

and

$$
T=\frac{128}{9} \frac{13+12 \cos 2 k l}{5897+6336 \cos 2 k l+567 \cos 4 k l} .
$$

Note that $R$ has a maximum for $k l=(2 \rho+1) \frac{\pi}{2}$. This is actually related to the fact that for the infinite approximant, these points correspond to the middle of gaps, as will be seen below (Subsection B). Indeed for a strip of infinite such (ordered) units parallel to the $11^{\prime}$-direction (i.e. in such a way as to have the output of one as the input of another), the total transfer matrix is an infinite product of elementary transfer matrices, and the corresponding (12)-element will diverge; a reflection coefficient that goes to infinity indeed signifies the presence of a gap.

What is however more interesting is the problem of a unit with a "flipped" internal structure (while keeping the external connectivities the same, to simulate the fact that the 
flipping is in the interior of only one unit cell and not in the external environment). This "flipped" system is shown in Fig.3-b. By going through the new matching equations and inverting the new $30 \times 30$ matrix we obtain the following exact results

$$
R=\frac{4(\cos k l)^{2}}{9} \frac{12781+8299 \cos 2 k l-180 \cos 4 k l-900 \cos 6 k l}{6821+6284 \cos 2 k l+255 \cos 4 k l-460 \cos 6 k l-100 \cos 8 k l}
$$

and

$$
T=\frac{128}{9} \frac{17+8 \cos 2 k l}{6821+6284 \cos 2 k l+255 \cos 4 k l-460 \cos 6 k l-100 \cos 8 k l} .
$$

From the structure of $(17)$ we see that for the special points $k l=(2 \rho+1) \frac{\pi}{2}$ (where the ordered system was found to have a maximum) the reflection coefficient vanishes rigorously. This vanishing is a very special situation that does not happen in the ordered system. It shows that if we put any finite number of flipped units in an otherwise ordered strip, the resistance will go down. Even more spectacular is the fact that, even an infinite strip of flipped units along this special symmetry direction will have a vanishing total reflection coefficient (due to the vanishing of the (12)-element of each transfer matrix, any product of these matrices, even of infinite of them, will have a (12)-element that will also be rigorously vanishing). The reflection coefficients (15) and (17) are plotted in Fig.4.

\section{B. Band Structure}

Let us now determine the band structure of the ordered infinite approximant network. We relate coefficients, through a phase $\phi_{1}$ or $\phi_{2}$ or $\phi_{1}-\phi_{2}$, for corresponding branches of unit cells that repeat in the three possible directions. An example is shown in Fig.5, showing connections through phases $\phi_{1}$ and $\phi_{2}$. The final result comes, after elimination of all coefficients, from a $12 \times 12$ determinant, and is

$$
\frac{2 m}{\hbar^{2}} E l^{2}=\left(\frac{1}{2} \arccos \left(\frac{\left(-3+4 \cos \phi_{1}+4 \cos \phi_{2}+4 \cos \left(\phi_{1}-\phi_{2}\right)\right.}{9}\right)\right)^{2} .
$$

This exact and analytically given band structure is plotted in Fig.6 (for fixed $\phi_{2}$ ). We see again the gap structure opening at regions where $\phi_{1}$ becomes a complex quantity. Once 
again we observe gaps in the origin, which is a manifestation of the Higgs mechanism: this is expected for any network; because even in the long-wavelength limit the space is full of "holes" and never homogeneous, resulting in the disappearance of Goldstone modes and the opening of a gap at the origin.

A contour-plot $E\left(\phi_{1}, \phi_{2}\right)$ of (19) (surfaces of constant energy) is also given in Fig.7. This shows the possible "Fermi surfaces", if the incidence energy is identified, as usually done, with the Fermi energy. (For free particles in full 2-d space we would of course have homocentric circles).

Note that the resonance upon flipping found above, is always in the middle of gaps of the ordered system. If the resonance is indeed a demonstration of extendedness of states (as it is in the case of a square network discussed earlier), one interpretation would be that new levels may be created in some gaps of the ordered system upon introduction of the phason-defect. This is in agreement with numerical findings for a 2 -d Penrose system ${ }^{4}$ in a tight-binding model.

We finally report that a calculation for a much bigger unit (that consists of a central unit cell together with all 6 neighboring unit cells, see Fig.8) gives the result also plotted in Fig.8. We note that a very deep minimum close to zero is also observed in the case that we only flip the central unit cell, whereas a very pronounced maximum appears in the ordered (unflipped) system.

\section{A 3-D PENROSE APPROXIMANT NETWORK}

We search for the above resonance phenomena in a more complex three-dimensional Penrose network, with a unit cell consisting of 4 rhombohedra of 2 types, and shown in Fig.9-a. It turns out that the problem is richer $^{7}$ than the corresponding 2 -d network, but 
in one case we indeed observe the same type of resonance upon flipping of the internal rhombohedra (see Fig.9-b), but again keeping the external connectivities the same. This result is shown in Fig.9-c, where the reflection coefficients of the ordered and the flipped system are compared.

\section{CONCLUSION}

We have found a vanishing reflection coefficient for a strip of flipped units in a 2-d and also in a 3-d network of wires for special incidence energies. The first obvious question is whether this type of resonance is experimentally detectable. With recent advances in microfabrication techniques extremely narrow wires can actually be manufactured. (Experiments in quasiperiodic superconducting networks ${ }^{10}$ have already been performed in the past). Alternatively, one can try the analogous acoustic experiments ${ }^{11}$ to observe the above phenomena. What is required for these types of resonances to be observed, is reflectionless reservoirs in the transverse channels, so that the "free-scattering" boundary conditions are satisfied.

From a theoretical point of view and in relation to quasicrystalline approximants, we have argued that the above resonances may be related to possible creation of extended states, upon flipping, in the gaps of the ordered system. If this is true, it may be seen as a possible mechanism of lowering the resistance with introduction of defects, a tendency that is in general agreement with real experiments ${ }^{1}$ on approximants and with numerical treatments ${ }^{4}$.

Finally, from an academic point of view, the above model systems are rare (if not unique) examples of exactly soluble band structures in high dimensionality. Furthermore, using these exact band structures as "input" one can go further to build a semiclassical 
dynamics $^{12}$ to study time-dependent propagation in these networks. Effects of disorder can also be studied through introduction of random potentials as already mentioned. Finally, introduction of a magnetic field is possible (and the problem is still soluble). This can address questions related to the Quantum Hall Effect ${ }^{13}$ in quasicrystalline approximants, a subject that is still open to investigation.

One of us (K.M.) acknowledges support from the European Union through the Human Capital and Mobility Program.

\section{REFERENCES}

1. For a review, see C. Berger in "Lectures On Quasicrystals", ed. F. Hippert and D. Gratias (Les Editions de Physique Les Ulis, 1994)

2. K. Moulopoulos and S. Roche, to be published in Int. J. Mod. Phys. B.

3. O. P. Exner and P. Seba, Rep. Math. Phys., 27, 7 (1989); J. E. Avron and L. Sadun, Phys. Rev. Lett., 62, 3082 (1989); Y. Avishai and J. M. Luck, Phys. Rev. B, 45, 1074 (1992)

4. T. Fujiwara, S. Yamamoto, and G. Trambly de Laissardiere, Phys. Rev. Lett., 71 (1993) 4166.

5. P. G. de Gennes, C. R. Acad. Sci. B 292, 279 (1981); S. Alexander, Phys. Rev. B 27, $1541(1983)$

6. D. C. Langreth and E. Abrahams, Phys. Rev. B, 24, 2978 (1981)

7. K. Moulopoulos, unpublished.

8. R. Landauer, Philos. Mag., 21 (1970) 863. 
9. P. Exner, Phys. Rev. Lett. 74, 3503 (1995)

10. B. Pannetier et al., Phys. Rev. Lett. 53, 1845 (1984); A. Behrooz et al., Phys. Rev. Lett. 57, 368 (1986); M. A. Itzler et al., Phys. Rev. B 47, 14165 (1993)

11. S. He and J. D. Maynard, Phys. Rev. Lett. 62, 1888 (1989)

12. N. W. Ashcroft and N. D. Mermin, "Solid State Physics", Holt-Saunders (1976), chapter 12

13. See M. Kohmoto, J. Phys. Soc. Jap. 62, 4001 (1993) 


\section{FIGURE CAPTIONS}

FIG.1 (a) Simplest single-vertex arrangement, with branch 1 being viewed as input, $1^{\prime}$ as output, and 0 as "transverse" branch. (b) Single vertex with two transverse branches 0 and $0^{\prime}$. (c) Elementary vertex for the square network problem. (d) Multi-vertex arrangement for a finite square network.

FIG.2 Movement of the band structure of a square network as $q_{2} l$ is varied. Solid curve corresponds to $q_{2} l=0$, Dashed-dotted to $q_{2} l=\frac{\pi}{2}$, and Dotted curve to $q_{2} l=\pi$. The two vertical lines show the positions of two special energies corresponding to $k l=\frac{\pi}{2}$ and $k l=\frac{3 \pi}{2}$. Note that these points always belong to a band. (Units $\frac{2 m}{\hbar^{2}} l^{2}=1$ are used).

FIG.3 (a) A low 2-d approximant network. There are three directions along which the unit cell is repeated; two of them are independent and are symbolized by the phases $\phi_{1}$ and $\phi_{2}$ (the third is then described by the phase $\phi_{1}-\phi_{2}$ ). A single unit cell is shown in the center, with arrows in the "outside branches" (which are determined by filling the plane with periodic repetition of this unit cell in all three directions). Single arrows signify "free-scattering" boundary conditions (see text, beginning of Section 4). 1 and 1' denote input and output branches (where reflections are allowed). (b) The result of flipping the interior of the central unit cell, but keeping the external environment the same.

FIG.4 Reflection coefficients, equ.'s (15) and (17), for the units shown in Fig.3(a) and 3(b). Unit 3(a) shows a local maximum, and unit 3(b) shows a vanishing at the special point $k l=\frac{\pi}{2}$.

FIG.5 Example of how to relate coefficients through $\phi_{1}$ and $\phi_{2}$, in order to determine analytically the band structure of an infinite network with the unit cell of Fig. 3-a.

FIG.6 Band structure of an infinite network with the unit cell of Fig. 3-a (for $\left.\phi_{2}=\frac{\pi}{2}\right)$. Gaps open at regions where $\phi_{1}$ becomes a complex number. 
FIG.7 Possible Fermi surfaces associated with the network with the unit cell of Fig. 3-a.

FIG.8 Reflection coefficients for a bigger central unit (shown on top left), consisting of 7 unit cells (one central and the 6 neighboring ones). For the ordered (unflipped) system (left) we observe a local maximum; for the system with a flipped central unit cell (right), we observe a deep minimum very close to zero $\left(R_{\min }=0.017\right)$, at $k l=\frac{\pi}{2}$.

FIG.9 (a) The unit cell of a low 3-d approximant. (b) The corresponding unit with "flipped" internal structure (but the same external environment). (c) The corresponding reflection coefficients showing a vanishing for the flipped system at the same special point $k l=\frac{\pi}{2}$. 